\title{
UMA QUESTÃO DE MÉTODO: DESAFIOS DA PESQUISA QUANTITATIVA NA SOCIOLOGIA
}

\author{
Ana Cristina M. Collares*
}

Resumo: Esse artigo discute a inserção das metodologias quantitativas nas ciências sociais sob várias perspectivas. De uma perspectiva epistemológica, abordamos a capacidade explicativa dos modelos estatísticos para as ciências humanas ou "soft sciences" e os desafios de combinar teoria e empiria para desvendar os mecanismos da ação social. De uma perspectiva histórica, mencionamos alguns estudos clássicos que se valeram de modelos quantitativos para desenvolver teorias nas ciências sociais, e discutimos o status atual da pesquisa quantitativa na área dada a maior disponibilidade de pacotes computacionais. Do ponto de vista prático, abordamos as dificuldades de se produzir pesquisas de qualidade com dados estatísticos, envolvendo dificuldades na amostragem, coleta e interpretação dos dados, e na validade e confiabilidade dos mesmos. Finalmente, questionamos as diferenças existentes entre a pesquisa qualitativa e a quantitativa nas ciências sociais, e as possibilidades da pesquisa quantitativa em termos de análise causal e de avaliação de políticas públicas.

Palavras-chave: Métodos em ciências sociais. Técnicas quantitativas de pesquisa. Ensino de metodologia na sociologia.

Abstract: This paper discusses the role of quantitative methodologies in sociological research under several perspectives. From an epistemological point of view it highlights the uses of statistics in humanities or "soft sciences" research, and the challenges to combine theory and empirical work in the search for social mechanisms. From a historical perspective, it presents some classical studies which used quantitative models to develop theories in social sciences, and the current status of social research, as well as issues related to the availability of specialized computational packages. From a practical point of view, the paper discusses issues concerning the production of high quality research with statistical techniques including problems with sampling, collecting and interpreting data, and problems with validity and generalizability of

* Professora da Universidade de Brasília - UnB, no Departamento de Sociologia - SOL. 
$|110|$

Uma questão de método: desafios...

conclusions. Finally, it presents some of the differences between quantitative and qualitative research to the production of causal analysis and public policy evaluation.

Keywords: Methods in social sciences. Quantitative techniques. Teaching sociological research methods.

\begin{abstract}
"Saiba o homem disso ou não, o alinhamento de seus problemas - como os formula e que prioridade atribui a cada um deles depende dos métodos, teorias e valores"
\end{abstract}

Wright Mills ${ }^{1}$

As pesquisas utilizando metodologias quantitativas vêm ganhando cada vez mais espaço na sociologia brasileira. A despeito desse fato, ainda há uma deficiência considerável no ensino desses métodos de pesquisa nos cursos de sociologia do país, com algumas exceções. Me apoio para fazer essa afirmativa tanto na minha experiência pessoal quanto na experiência do sociólogo Ignacio Cano, expressa em artigo recente em que ele constata que "as ciências sociais brasileiras privilegiam a teoria em detrimento da pesquisa, promovendo um conhecimento antes erudito do que técnico" (Cano, 2012, p. 111)2, e ainda que "dentro da teoria, a primazia absoluta é conferida aos clássicos e à grande teoria social, mais do que a Teorias de Alcance Intermediário (Merton) ou microteorias apropriadas a campos concretos". A consequência mais imediata dessa realidade, segundo Ignácio Cano, é que essa "predominância da teoria sobre a prática milita contra a importância outorgada ao método no ensino de sociologia" (Cano 2012, p. 112). Com base nesses dois pressupostos - o da crescente presença das metodologias quantitativas na pesquisa sociológica brasileira, e o da endêmica resistência dos acadêmicos dessa disciplina a dar mais espaço ao aprendizado de métodos e técnicas

\footnotetext{
${ }^{1}$ Mills, W. A imaginação Sociológica. Rio de Janeiro: Zahar, 1982.

${ }^{2}$ Há também uma interessante discussão sobre o uso dos métodos quantitativos e qualitativos na ciência política brasileira em Soares, 2005.
} 
de pesquisa nos cursos de sociologia do País ${ }^{3}$ - pretendo discutir nesse artigo algumas das vantagens, dos desafios e problemas do uso desses métodos no contexto da sociologia, e especificamente no contexto da sociologia brasileira.

A expressão "metodologias quantitativas" representa aqui os métodos de análise que empregam técnicas estatísticas e fazem uso de amostras representativas de determinadas populações. Os dados que alimentam essas pesquisas atualmente são, em geral, coletados ou pelo próprio pesquisador, ou por órgãos públicos e empresas privadas, principalmente em questionários do tipo survey ${ }^{4}$ (enquete, sondagem) e armazenados em planilhas eletrônicas. Uma das justificativas do uso de tais metodologias é a maleabilidade que estas proporcionam para responder a questões sociológicas e realizar inferências causais nas ciências humanas ao utilizar grande número de casos e modelos estatísticos que podem ser facilmente calculados pelos softwares específicos, os quais testam hipóteses com sofisticadas equações. As técnicas mais utilizadas nas ciências sociais dentro desses parâmetros são as análises de regressão linear e não linear em todas as suas modalidades. Os pacotes computacionais se desenvolveram enormemente nas últimas décadas, aumentando a velocidade de processamento dos dados, o número de variáveis que pode ser empregado nos modelos e o tamanho das amostras de casos para análise. Isso revolucionou a análise nas ciências sociais, e essa mudança teve consequências positivas e negativas, as quais torna-se imprescindível explicitar para que a crescente presença de tais metodologias no contexto nacional venha a enriquecer, e não a empobrecer, o aprendizado e a prática da pesquisa sociológica no Brasil.

${ }^{3}$ Os alunos têm aprendido essas técnicas principalmente em cursos de especialização tais como o curso de métodos quantitativos - MQ que vem sendo oferecido anualmente na UFMG, ou no exterior.

${ }^{4}$ A pesquisa do tipo survey significa "a obtenção de dados ou informações sobre características, ações ou opiniões de determinado grupo de pessoas, indicado como representante de uma população alvo, por meio de um instrumento de pesquisa, normalmente um questionário". (Freitas et al. 2000, p.105) 
$|112|$

Uma questão de método: desafios...

O método de análise tem sido uma preocupação constante dos sociólogos, e constante foco de discussão acadêmica dentro da sociologia. O cientista social está constantemente refletindo sobre suas metodologias de trabalho, devido à constante necessidade de validar o conhecimento que produz dado o próprio "compromisso da sociologia, já na sua origem, com os problemas sociais que afetam os homens que vivem em sociedade, e que desafiam a imaginação dos pesquisadores", ou seja, do conflito entre subjetividade e objetividade envolvida na análise de seus objetos (Martins, 2010 p.22). Essa necessidade de validação se dá tanto mais pela diversidade de controvérsias em torno da natureza científica do conhecimento social do que propriamente pelas peculiaridades de seu objeto de análise. As ciências sociais teriam, citando Schwartzman (1971 apud Freitas, 2010), "a desagradável inabilidade [...] de se comportar corretamente como as outras ciências", ou seja, de produzir análises dentro do chamado "paradigma metodológico causal" (Marino, 2012) que é o paradigma canônico da inferência científica. Mas em que consiste esse paradigma canônico e como ele se reflete na pesquisa em sociologia e na validação do conhecimento sociológico?

O sociólogo Arthur Stinchcombe, no livro Constructing Social Theories (1968) dá uma descrição do modo de se fazer inferências científicas nas ciências sociais que, segundo ele, "forma a base comum das ciências em geral" (para uma discussão desse modo canônico de inferência científica e sua penetração nas ciências sociais, sugiro a discussão realizada em Freitas e Collares, 2001). ${ }^{5}$

\footnotetext{
5 Ao fazer essa discussão, não estou negando ou negligenciando propositalmente a existência de outras formas de realizar a empresa sociológica tanto do ponto de vista prático como teórico. A discussão sobre os desafios de novas formas de análise sociológica, sobre a sociologia intuitiva, crítica ou baseada em concepções pragmáticas de ciência ou em concepções que fogem aos cânones científicos já tem sido feita de maneira exaustiva. O objetivo desse trabalho é primordialmente chamar a atenção dos pesquisadores em sociologia para os problemas que podem advir tanto de uma ênfase excessiva nas vantagens das metodologias quantitativas quanto da reificação e distinção de abordagens quantitativas e qualitativas como entidades separadas. Assumo
} 
De acordo com Stinchcombe, a construção de teorias científicas requer que o pesquisador tenha em mente os requerimentos lógicos segundo os quais a teoria pode ser testada contra os fatos. A inferência científica, continua ele, começa com um enunciado teórico, "um elemento de uma teoria que diz que classe de fenômenos vai se conectar de um certo modo com uma outra classe de fenômenos," (Stinchcombe,1968, p. 15). "Desse enunciado teórico nós podemos derivar, por dedução lógica e definições operacionais de conceitos, um enunciado empírico." (1968, p.16) Esse enunciado por fim vai permitir a tradução da teoria em eventos passíveis de ser observados que vão testar se as relações causais ou correlacionais estabelecidas no enunciado teórico são falsas ou se nosso enunciado vai se tornar mais robusto (credible em inglês no original), proporcionando um avanço no conhecimento sobre tal ou qual assunto. O método de pesquisa, seja ele qual for, instrumentaliza o teste empírico de teorias e hipóteses (Cano, 2012), e sua explicitação exaustiva constitui ferramenta poderosa no processo de validação da análise no contexto das ciências sociais.

A sociologia se caracteriza por uma multiplicidade de métodos, porém, no presente, podemos dizer que existe uma separação, no discurso e na prática dos cientistas sociais, entre métodos quantitativos e métodos qualitativos de pesquisa. Essa separação constitui um divisor de águas na sociologia, separando sociólogos não apenas pela adesão a determinados métodos, mas também pela associação desses métodos a determinadas teorias e mesmo ideologias (Cano 2012). Nesse trabalho, pretendo discutir alguns dos males que podem surgir do uso indiscriminado de técnicas quantitativas nas ciências sociais. Acredito ser extremamente válido que aprendamos com a experiência de departamentos de sociologia ao redor do mundo onde o ensino e o uso dessas metodologias já estão consolidados. Nesse sentido pretendo também demonstrar que, se por um lado a pesquisa

nesse trabalho a abordagem científica descrita por Stinchcombe (1968) como recurso heurístico para atingir os objetivos propostos, sem renegar a validade de outras formas de produção do conhecimento em sociologia. 
| 114 |

Uma questão de método: desafios...

quantitativa tem um papel fundamental na prática da inferência científica dentro das ciências sociais, pois possibilita um tratamento sistemático e generalizável do dado empírico e confere robustez às afirmativas feitas por cientistas sociais, por outro lado, essa divisão entre métodos qualitativos e quantitativos é prejudicial à prática da pesquisa nessa disciplina.

\section{Teoria e Empiria}

Antes, de falar da distinção entre métodos qualitativos e quantitativos, é imprescindível mencionar uma outra separação, ao meu ver, de caráter ainda mais corrosivo para a sociologia. Tratase da distinção entre teoria e empiria, que se faz notar em muitos trabalhos sociológicos, sobretudo de pesquisadores iniciantes, mas também em trabalhos de sociólogos experientes. Acontece com alguma frequência em trabalhos na área de sociologia, que o pesquisador ou constrói todo um argumento descritivo com base em dados observados sem que haja uma fundamentação teórica para a observação, ou falha em utilizar a teoria ${ }^{6}$ para orientar a tomada de conclusões sobre os dados observados.

Em geral, e estou ecoando aqui a opinião de Aage Sorensen (1998), a pesquisa sociológica focada no trabalho empírico tem sido pobre em teoria, ao mesmo tempo que não é informativa o suficiente para alimentar o progresso teórico. Faltam não apenas uma ênfase maior na construção de teorias de médio alcance (Merton, 1968), uma prática pouco discutida no ensino da sociologia no Brasil, como menciona Cano (2012), mas também em pesquisas empíricas fundamentadas em enunciados teóricos bem embasados, ou pesquisas capazes de produzir novas teorias. Nos contextos onde o ensino de métodos quantitativos de pesquisa em ciências sociais é consolidado, tais como o contexto americano, essa

${ }^{6}$ Teoria, nesse caso, entendida como mais do que um simples teste de hipóteses estatístico, ou seja, como uma explicação dos mecanismos ou do sentido que orienta(m) a ação social. 
separação entre teoria e empiria vem ocorrendo, sobretudo porque o grande desenvolvimento desses métodos coloca a pesquisa sociológica quase que como um ramo da estatística.

Essa linha de pesquisa com forte ênfase nos modelos estatísticos de análise se concentra muitas vezes nas conclusões retiradas desses modelos, ou seja, na "significância" ou não da relação entre variáveis baseada no teste estatístico de hipóteses, negligenciando a pesquisa dos mecanismos que levam aos processos sociais observados. Sorensen (1998), e faço minhas as suas palavras, afirma que não é possível usar métodos estatísticos para analisar as relações entre as variáveis sem especificar o modelo matemático dessas relações. Porém, muitas das pesquisas sociológicas chamadas quantitativas empregam simples modelos aditivos de regressão que pressupõem relações lineares entre as variáveis. Esses modelos, apesar de simples, ainda assim necessitam da especificação do mecanismo através do qual as variáveis se relacionam, e esse fato muitas vezes escapa ao pesquisador social, especialmente àquele cujo treinamento enfatizou os modelos estatísticos de uma forma dissociada de sua significação teórica e empírica. Esse pesquisador emprega mecanicamente variáveis como sexo, raça e renda familiar como "controles estatísticos" em sua análise, ausentando-se da discussão sobre para que tipo de dados e de relações formuladas teoricamente seus modelos estatísticos são bons instrumentos de teste. Quando o mecanismo explicativo das relações sociais investigadas é fornecido simplesmente pelo modelo estatístico, a teoria se dissocia da evidência (Sorensen, 1998).

A "matematização" da realidade em um sentido mais amplo, ou seja, a formulação de funções matemáticas que definam as relações entre variáveis empíricas com base em alguma teoria sociológica, não é em geral ensinada nos cursos de sociologia, com raras exceções. Muitas vezes o estudante em treinamento e mesmo alguns pesquisadores mais experientes se perdem na especificação do modelo estatístico, de quais variáveis vão usar como "controles" ou variáveis "dependentes", de qual método de regressão vão empregar, e se esquecem de que o objetivo primordial do modelo 
| 116 |

Uma questão de método: desafios...

estatístico " $\mathrm{p}$ " é submeter algum modelo teórico " $\mathrm{q}$ " anteriormente formulado ao teste da observação. Nesse teste, tal como o descreve Stinchcombe (1968), estão previstos a validação ou o falseamento de mecanismos micro ou macro-estruturais construídos pelo pesquisador através de seus enunciados teóricos para explicar os fenômenos sociológicos estudados.

\section{A crescente complexidade dos modelos estatísticos de análise}

Um outro problema da ênfase em análises estatísticas, o qual compromete a conexão entre a teoria e a empiria e a busca por mecanismos explicativos da ação e da interação social é a atração que exerce sobre o investigador a existência de modelos de análise estatística cada vez mais complexos. Essa atração chega ao ponto de determinados periódicos se recusarem a publicar artigos extremamente ricos do ponto de vista teórico, mas que "não possuem uma amostra representativa", ou que "não utilizaram métodos de imputação múltipla para os poucos casos ausentes existentes nos dados" e assim por diante. A questão é que essa crescente atração e mesmo exigência pela complexidade estatística na análise pode comprometer o próprio processo de validação empírica do conhecimento, obrigando o pesquisador a adaptar os dados aos modelos de forma muitas vezes inadequada ao próprio modelo, resultando em perda da capacidade explicativa do mesmo. Os processos sociais são fenômenos complexos. É exatamente por isso que a sociologia procura abstrair determinadas características desses processos buscando simplificá-los para compreender os mecanismos que atuam na sua produção. "A tarefa da teoria é simplificá-los e caracterizá-los em termos de seus elementos essenciais" (Sorensen, 1998, p. 242). Assim, um simples cruzamento de variáveis pode ser suficiente para simplificar os processos sociais facilitando o desenvolvimento de teorias. E muitos sociólogos trabalham com essas técnicas simples tais como a análise descritiva e as tabelas de contingência com excelentes resultados para a validação de seus enunciados teóricos. Modelos estatísticos 
complexos devem entrar em cena na investigação apenas após cuidadosa análise exploratória dos dados. Essa exploração inicial pode ser feita usando técnicas tais como o cruzamento de variáveis, a análise de correlação e gráficos de dispersão, pois aí as relações entre as variáveis que vão entrar nos modelos ficam mais claras.

Quando os estudantes de sociologia, especialmente aqueles em treinamento para realizar pesquisas empíricas, partem imediatamente para o uso de modelos estatísticos complexos antes de realizar uma boa exploração dos seus dados, e as análises passam a se limitar a informes sobre a significância estatística e a magnitude de coeficientes de regressão. Isso ocorre frequentemente em cursos de métodos quantitativos quando o entusiasmo pela técnica pode levar o aluno a pular etapas na investigação dos dados. Essa forma de análise se mostra, como já foi dito, pobre em teoria, e não importa nesse caso se a regressão estatística utilizada foi simples, logística ou mesmo hierárquica, se foi usado o método de mínimos quadrados ou o método matricial de cálculo, e assim por diante. O tipo de cálculo utilizado é em geral definido pelo pacote computacional disponível, e os resultados são tomados como conclusões após a obtenção dos coeficientes resultantes do método. As explicações assim obtidas às vezes passam a ser classificadas como "modelos analíticos", separadas dos "modelos teóricos", e a teoria fica desvinculada das evidências empíricas (Sorensen, 1998).

Inegavelmente, o desenvolvimento dos modelose dos pacotes computacionais que os calculam possui inúmeras vantagens para a pesquisa sociológica. Como algumas consequências positivas desse desenvolvimento menciono a crescente facilidade que as pesquisas sociológicas encontram em lidar com um número de dados cada vez maior, a maior velocidade com que os resultados dos modelos de análise são obtidos, a facilidade do uso e a maior confiabilidade dos cálculos, e a combinação de dados de natureza longitudinal, favorecendo a descoberta de padrões sociais. Porém, junto com essas facilidades vêm a primazia do dado e do cálculo sobre o raciocínio sociológico e o uso indiscriminado de modelos estatísticos sem a correta noção do que estes representam, pois são 
| 118 |

Uma questão de método: desafios...

calculados pelos computadores e o pesquisador perde de vista que tipo de relações esses cálculos estão representando.

As primeiras grandes pesquisas quantitativas se utilizaram de centenas de casos e diversas variáveis, mas nessas pesquisas a metodologia continha implícita a teoria que procurava testar. O Relatório Coleman (1967), por exemplo, utilizou dados sobre milhares de estudantes de escolas americanas, procurando medir o efeito das escolas sobre o desempenho dos alunos através da análise da variância relacionada a testes de proficiência. A análise de variância é um modelo simples que não estipula relações complexas entre variáveis, mas apenas indica que porção da variação de uma variável dependente, nesse caso a proficiência, está conectada à variação de variáveis independentes, como por exemplo o número de livros e computadores existentes em uma escola. Outro exemplo é o uso da análise de trajetórias por Blau e Duncan no livro The American Occupational Structure (1967), que praticamente introduziu a análise de regressão nas ciências sociais para muitos pesquisadores (Sorensen, 1998). Porém, nesse caso, apesar de os coeficientes mostrados como resultado da análise serem equivalentes a coeficientes de regressão, a especificação dos mecanismos teóricos estava intrínseca no modelo estatístico. Isso porque na análise de trajetórias os coeficientes de regressão são calculados especificando a maneira como as variáveis se relacionam entre si através de diversas trajetórias, que combinadas formam diversas equações de regressão. Essa maneira de representar o modelo favorece a construção teórica e a especificação dos mecanismos através dos quais algumas variáveis influenciam outras, como se fosse um "mapa causal". Porém essa é uma técnica atualmente pouco utilizada nas ciências sociais (ela é mais comum nas pesquisas da psicologia social nos Estados Unidos, por exemplo).

A forte ênfase na observação empírica e no estudo das micro-relações sociais, tanto no período entre guerras como no pós-guerra, favoreceu a hegemonia de modelos estatísticos cada vez mais complexos, especialmente na sociologia americana. A existência de bons trabalhos oriundos desse período, ricos no 
estabelecimento de mecanismos teóricos ainda que do ponto de vista da micro-sociologia e das teorias de médio alcance (vide os trabalhos quantitativos baseados nas teorias da desorganização social, nascidas no contexto da Escola de Chicago descritos em, por exemplo, Becker, 1996) confirma que o estabelecimento de mecanismos teóricos pode ser representado na análise quantitativa de diversas formas, que não necessariamente implicam em complicadas relações multivariadas. Entretanto, conhecer essas técnicas, ainda que o pesquisador opte por não utilizá-las, é um desafio para futuros sociólogos, tanto do ponto de vista de aprimorar e abrir novas possiblidades para o próprio trabalho quando da necessidade de compreender o trabalho de outros. Não podemos cair naquilo que descreve Sorensen como "uma das ironias do treinamento pós-graduado [em sociologia] contemporâneo", ou seja, "que a teoria algumas vezes se torna um refúgio para estudantes que não sabem ou não gostam de aprender estatística" (Sorensen, 1998, p.242).7

\section{Os sociólogos e a análise de políticas públicas}

A análise de políticas públicas é um campo da sociologia que muitas vezes envolve o estabelecimento de relações causais. Esse é um grande desafio para a pesquisa quantitativa nas ciências sociais. A ainda corrente escassez de sociólogos proficientes em análises quantitativas no contexto brasileiro acaba afastando da prática um possível e interessante campo de trabalho, no qual a formação geral do sociólogo permitiria a este prestar serviços de excelência. Ignácio Cano, ao mencionar o crescente número de pós-graduações em políticas públicas surgindo no Brasil, afirma que esses novos diplomas tiveram que ser criados porque não foi possível abordar essa questão de dentro dos departamentos

\footnotetext{
${ }^{7}$ Do inglês no original: "One of the ironies of contemporary graduate training is that theory sometimes becomes the refuge for students who are unable or unwilling to learn statistics".
} 
| 120 |

Uma questão de método: desafios...

de sociologia. Assim, continua ele, "se por um lado essas novas graduações são um triunfo para os que pretendemos modificar o paradigma convencional [das Ciências Sociais no Brasil], por outro acabam confirmando indiretamente a ideia de que um especialista em políticas públicas não é um cientista social". (Cano, 2012 p.114)

$\mathrm{O}$ "efeito" de uma variável sobre outra não necessariamente significa uma relação causal entre as duas variáveis, muito menos diz algo a respeito da direção dessa causalidade. Apesar de que a análise de trajetórias, por exemplo, indica uma direção causal para os efeitos, e nas análises de regressão existem as variáveis "dependentes" e "independentes", esta direção dos efeitos dada pelos modelos estatísticos é antes uma especulação teórica do que um teste empírico da direção de causalidade. O suporte teórico torna-se especialmente importante aqui, para orientar o investigador na definição de quais são essas variáveis dependentes e independentes. A despeito da relevância de tais análises, muitos cientistas acreditam que o uso de experimentos controlados é a única maneira segura de estabelecer relações causais, e os cientistas sociais ao redor do mundo não fogem a esse padrão. Recente relatório americano sobre as pesquisas educacionais envolvendo grande número de cientistas sociais (Schneider at. al. 2007) concluiu que o experimento controlado é praticamente o único meio seguro de obter relações causais, e na falta deste os chamados quasi-experiments ou quase-experimentos, que são as técnicas usadas no manejo de dados observacionais, em geral baseados em uma amostra populacional, que permitem a comparação entre diferentes "tratamentos". Estes últimos devem ser usados com cautela, afirmam os autores, pois incorrem em vários riscos que podem trazer imprecisão aos resultados. Nas ciências sociais, experimentos controlados são ainda muito mais complicados de se realizar do que nas ciências exatas. Em primeiro lugar, as questões éticas são muito complexas. Por exemplo, ao analisar a implementação de um método que poderia ajudar escolas públicas, como escolher aquelas que receberão a implementação do método ou "tratamento" e aquelas que receberão o "placebo", ou seja, o grupo de controle? Como evitar chamado efeito "spill over" 
(grosso modo, "derramamento"), ou seja, que alguns professores da instituição recebendo o tratamento contem aos professores de instituições que estão no grupo de controle sobre o novo método, fazendo com que algo deste seja usado nas escolas de controle "derramando" o tratamento e comprometendo os resultados do experimento? Outro problema sério que pode surgir em um experimento social é o atrito, ou seja, aqueles participantes que aderem inicialmente ao projeto e depois o abandonam. Como, dado esse atrito, avaliar o "antes" e o "depois" de um experimento se o grupo inicial não é o mesmo do final? Finalmente, vale observar que para ter poder suficiente de detectar um efeito, muitas vezes um experimento tem que contar com grande número de casos (o cálculo do número de casos necessários para detectar um efeito é chamado de força ou power da análise). Isso às vezes encarece o procedimento de tal maneira que torna quase proibitivo planejar experimentos sociais.

Uma das alternativas ao experimento controlado é analisar os chamados "experimentos naturais". Por exemplo, a loteria usada para selecionar alunos em algumas charter schools ${ }^{8}$ nos Estados Unidos, ou a que ocorre em algumas escolas públicas no Brasil, sorteando os candidatos ao invés de instituir uma prova de seleção. Nesse caso supõe-se que os alunos que não ganham o sorteio, mas participaram do mesmo têm características semelhantes aos que foram sorteados, e podem servir como grupo de controle tal qual se os participantes do experimento tivessem sido sorteados aleatoriamente para entrar nos grupos de tratamento e controle. Esse tipo de situação constitui o que se chama de experimento natural, e oferece grandes oportunidades para a análise causal. Ainda assim, esses experimentos naturais são raros. Existem outros métodos que buscam simular os efeitos obtidos com experimentos, como a análise de escores de propensão (propensity scores), o uso do design de descontinuidade de regressão (regression descontinuity design), o uso de variáveis instrumentais, entre outros

\footnotetext{
${ }^{8}$ Um tipo de escola americana que recebe dinheiro público, mas tem mais
} flexibilidade na administração do que as escolas públicas tradicionais. 
| 122 |

Uma questão de método: desafios...

(para esse tipo de técnicas ver, por exemplo, Scheider et al. 2007). Porém, é necessária muita cautela com as conclusões derivadas de todos esses métodos, pois experimentos sociais não podem ser reproduzidos da mesma forma que nas ciências exatas, e os fatores contextuais em torno do experimento podem causar desvios nos resultados com muito mais frequência do que nas ciências naturais devido à imprevisibilidade do fator humano.

Com o que foi dito acima, os analistas de políticas públicas (entre eles os sociólogos) se vêm sujeitos a lidar com dados criados "ex-post", ou seja, depois da implementação das políticas, e muitas vezes produzidos sem o objetivo específico de favorecer a análise destas. Esses dados poderão ser então analisados com métodos que incluem as análises de regressão, e podem até mesmo oferecer boas respostas sobre os efeitos de políticas públicas, especialmente se a amostragem foi bem feita. Porém um bom conhecimento das técnicas de análise causal, dos quase-experimentos e das demais técnicas de análise quantitativa são imprescindíveis para tirar o melhor proveito dos dados disponíveis. Investigações nessa linha auxiliam não apenas na análise de políticas públicas, mas em diversas outras questões sociológicas.

Vale repetir que métodos de análise empírica não experimentais e as técnicas estatísticas conectadas a eles ${ }^{9}$ possuem uma série de limitações que, se não levadas em conta, podem levar a uma supervalorização das conclusões oferecidas pelos mesmos. Minimizar os problemas e levar em conta as limitações das técnicas são importantes desafios das análises quantitativas na sociologia, que dependem de um bom treinamento nesse tipo de análise. A seguir discorro brevemente sobre algumas dessas limitações.

\footnotetext{
9 Ignacio Cano diferencia entre métodos e técnicas afirmando que esses conceitos são muitas vezes usados como sinônimos mas podem ser diferenciados, sendo o conceito de método mais ligado à epistemologia, e por consequência mais abrangente. "Métodos seriam estratégias de produção de conhecimento científico, incluindo a geração e a validação de teorias. Técnicas seriam formas padronizadas de coleta e análise de dados, com a mesma finalidade, a de produzir conhecimento válido." (2012, p.107)
} 


\section{A qualidade dos dados na análise quantitativa}

Uma das limitações do uso de metodologias quantitativas em sociologia é a representação de conceitos teóricos através de dados empíricos estatisticamente manipuláveis, ou seja, a instrumentalização da teoria. Conceitos teóricos tais como capital social e capital cultural, por exemplo, encontraram diversas formas de representação na pesquisa quantitativa, mas é praticamente impossível definir se o dado está representando adequadamente o conceito desejado ou não. Para se ter uma ideia, o conceito de capital cultural já foi operacionalizado de inúmeras maneiras, tais como "conhecimento da alta cultura" (DiMaggio e Useem 1978), ou "currículo de escolas de elite" (Cookson e Persell 1985), ou "alcance educacional" (Robinson e Garnier 1985), ou ainda "capacidade de realizar tarefas de modo culturalmente aceitável" (Gouldner $1979)^{10}$. Outros conceitos muito usados em pesquisas sociais e que são de difícil representação empírica são os conceitos subjetivos tais como autoestima, anomia, autodeterminação, entre outros, que acabam por fazer parte da "heterogeneidade não-mensurável" do modelo, e cujos desvios ou vieses são colocados no coeficiente do "erro" da equação de regressão. Diversos métodos de controle dessa heterogeneidade existem na pesquisa quantitativa, tais como o modelo de Heckman (1979), os modelos de classes latentes, o método de mínimos quadrados em dois estágios etc., mas nenhum deles teria a capacidade de eliminar completamente o viés causado pela impossibilidade de medir determinada variável importante para a teoria que está sendo testada.

Ainda nesse tópico, vale alguns outros exemplos que demonstram a dificuldade de operacionalizar ou mensurar variáveis utilizadas nas ciências sociais. Por exemplo, esquemas de classificação de sentimentos e opiniões de entrevistados, tais

\footnotetext{
${ }^{10}$ Lareau e Weininger (1985) discutiram a operacionalização na pesquisa quantitativa do conceito de capital cultural, citando estes e outros artigos que fizeram pesquisas empíricas utilizando o conceito de capital cultural, e realizando uma interessante crítica sobre o conteúdo contido em cada conceito em opoisição ao conceito teórico derivado de Pierre Bourdieu.
} 
como a classificação de uma opinião segundo a escala $L_{i k e r t}{ }^{11}$, são escolhas de certo modo arbitrárias, que devem ser bem justificadas para fazer sentido para outros que não o próprio pesquisador. Um outro exemplo de transformação de uma variável que pode ocorrer de maneira arbitrária é a definição do ponto de corte para transformar uma variável contínua ou uma variável com muitas categorias em uma variável dummy ${ }^{12}$. Se esse ponto de corte não estiver bem justificado na pesquisa, e outros possíveis pontos de corte não forem testados, a mesma variável pode produzir resultados muito diferentes dependendo de como foi "cortada".

A necessidade de se obter uma amostragem adequada para a análise, do ponto de vista da representatividade da população em estudo, é outro fator limitador da pesquisa quantitativa. De uma boa amostragem depende a confiabilidade e a validade dos dados, bem como que tipo de conclusões que podemos tirar da análise destes e que tipo de métodos podemos usar. Por exemplo, os modelos hierárquicos de regressão, ou modelos de multinível são frequentemente utilizados pelos pesquisadores em qualquer tipo de amostra, sendo que o ideal é que esses modelos sejam utilizados apenas quando a amostragem foi feita de modo estratificado pelo nível de "aninhamento" (nesting) do modelo. Assim, quando usamos um modelo de multinível para analisar alunos dentro de escolas, ou empresas dentro de municípios, primeiro temos que amostrar as escolas, ou os municípios, e dentro destes amostrar alunos e ou empresas que sejam representativos de cada escola/ município amostrado. Quando apenas os alunos e as empresas são amostrados, utilizar um segundo nível de análise (escolas, municípios) em geral não é adequado, pois o risco de os alunos/ empresas não serem representativos de cada escola/município é grande, comprometendo a análise. ${ }^{13}$

\footnotetext{
${ }^{11}$ Escala psicométrica muito usada em questionários de pesquisas de opinião, possuindo em geral 5 pontos.

${ }^{12}$ Uma variável binária cujos valores são 0 ou 1.

${ }^{13}$ Para saber mais sobre os modelos hierárquicos ou de multinível, sugiro a leitura de Raudenbush and Bryk (2002) e, em português, Natis (2001).
} 
A estratificação da amostra tem que ser levada em conta não só nesta, mas em diversos outros tipos de análise. A amostra da Pesquisa Nacional por Amostragem de Domicílios - PNAD (IBGE) - , por exemplo, possui vários níveis de estratificação (estado, região metropolitana, etc.), e nos anos mais recentes os bancos de dados da PNAD disponibilizados aos pesquisadores possuem variáveis que permitem levar essa estratificação em conta nas análises de regressão para evitar ou reduzir vieses na análise, especialmente quando são usados alguns tipos de pesos. Existem modelos estatísticos que levam em conta o perfil amostral dos dados e possibilitam o uso dessas variáveis para corrigir possíveis desvios nas previsões derivadas desses dados ${ }^{14}$. Finalmente, o uso de pesos também tem que ser levado em conta de acordo com a amostra, para garantir a generalização dos resultados para uma determinada população.

Um problema também comum na construção de amostras representativas é a omissão dos entrevistados em fornecer determinadas informações tais como a renda pessoal, gerando grande número de casos ausentes e enviesando a amostragem no que se refere à variável renda. Vieses podem ocorrer também devido à existência de grupos que ficam excluídos da amostra por omissão de informações (underreporting). Por exemplo, estudos sociológicos sobre violência e pequenos crimes podem usar como fonte de dados o registro dos crimes nas delegacias, porém a maioria dos crimes menores ocorridos em uma população não são registrados pelas vítimas, prejudicando o cálculo de representatividade da amostra (ver, por exemplo, Myers, 1980, e Pinto e Ribeiro, 2008). Conclusões retiradas desses dados podem ser enviesadas pelo desconhecimento das reais taxas de crime em uma população. Uma melhor maneira de pesquisar a criminalidade ou a violência então poderia ser com o uso das pesquisas de vitimização, que

${ }^{14}$ Ver, para uma descrição dessas variáveis na PNAD, o trabalho de Silva, P. L. N. et al., 2002. 
$|126|$

Uma questão de método: desafios...

entrevistam os indivíduos diretamente calculando as proporções de ocorrência de determinados crimes na população. ${ }^{15}$

Independente da técnica de amostragem, a maneira como o dado é coletado também pode interferir na informação que ele está transmitindo. Em geral, os pesquisadores lidam com dados de "segunda mão", ou seja, coletados por outros, ou realizadas por centros de pesquisa onde vários grupos de pesquisadores são responsáveis por diferentes partes de um questionário. Grandes bases de dados com amostras representativas são muito caros e trabalhosos e dificilmente são produzidas por pesquisadores isolados. Como exemplos desses dados de segunda mão podemos citar o Censo demográfico e as pesquisas periódicas como as Pesquisas Nacionais por Amostragem Domiciliar (PNADs), feitos pelo IBGE no Brasil, ou surveys menores como a Pesquisa da Região Metropolitana de Belo Horizonte (PRMBH), realizada por vários grupos de pesquisa da UFMG, etc. (Aguiar, 2007) Nesse caso, informações mais subjetivas a respeito da interação entre o informante, o entrevistador e o assunto abordado, do tipo "quanto tempo o informante demorou para responder à questão?", "houve hesitação na resposta?" etc., acabam se perdendo, a não ser que se planeje capturar esses aspectos da entrevista de antemão, como foi feito na mencionada PRMBH (Simões e Pereira, 2007). Mesmo quando a coleta dos dados é feita pela mesma pessoa que vai analisá-los, muitas vezes essas nuances não são levadas em consideração. Existem algumas técnicas que podem ser utilizadas em questionários de survey que buscam captar algumas particularidades ou subjetividades do respondente, tais como a utilização de probes ou questões que são colocadas logo depois de determinada resposta ao questionário a fim de testar com mais profundidade o conhecimento do respondente a respeito do que foi perguntado. As questões em um questionário podem ser manipuladas pelo pesquisador de diversas formas no momento da

\footnotetext{
${ }^{15}$ Entre 2010 e 2012 foi realizada a primeira pesquisa nacional de vitimização no Brasil, cujo sumário executivo pode ser lido em: http://www.crisp.ufmg.br/ wp-content/uploads/2013/10/Sumario_SENASP_final.pdf
} 
aplicação a fim de obter respostas mais exatas, como por exemplo modificando o enunciado das questões, alterando o número de categorias a serem selecionadas ou introduzindo categorias intermediárias tais como "nem um nem outro", etc. Certas perguntas também lidam com assuntos "sensíveis" e vão ser mais confiáveis se o questionário for anônimo, por exemplo. Saber que o dado, seja ele primário ou secundário, não é uma entidade neutra mas está sujeito a todos esses tipos de variações e interferências, e procurar reduzir o impacto dos fatores não controlados (subjetividade do respondente, condições de entrevista, contexto de aplicação, comportamento do aplicador etc.) nas informações selecionadas para uma pesquisa são desafios da pesquisa quantitativa. ${ }^{16}$

\section{A escolha do método de pesquisa}

A concatenação entre a teoria e a evidência empírica está intrinsecamente ligada à escolha do método de análise em qualquer pesquisa, e o uso adequado desse método responde por grande parte da qualidade do trabalho. Em termos das técnicas quantitativas, com as facilidades computacionais modernas o pesquisador vai ser quase sempre tentado a assumir relações lineares entre todas as variáveis utilizadas na pesquisa, e "normalizar"17 ou transformar de alguma outra forma as variáveis que não possuem uma distribuição padrão para garantir essa linearidade. As técnicas de regressão múltipla do tipo "stepwise" são as mais frequentemente adotadas. Estas operam em geral com o pesquisador adicionando à equação de regressão todas as variáveis de "controle" ou variáveis independentes possíveis e

\footnotetext{
${ }^{16}$ Ver Pereira e Simões (2010) e Simões e Pereira (2007) para uma discussão a respeito da subjetividade do respondente e outros fatores que podem causar viés nas respostas dadas a questionários de pesquisas tipo survey.

${ }^{17}$ Transformar uma variável de tal modo que sua distribuição se assemelhe à da curva normal, por exemplo, tomando o logaritmo natural de uma distribuição de renda.
} 
eliminando paulatinamente aquelas que não são "significativas". Esse tipo de procedimento é um dos maiores responsáveis pela total separação entre a pesquisa empírica e a teoria. $\mathrm{O}$ pesquisador simplesmente não se debruça sobre os dados com uma hipótese teórica dos mecanismos de relacionamento entre as variáveis, mas assume que todas as relações são lineares (ou possuem alguma outra forma esperada, tal como na análise logística de variáveisresposta binárias) e espera que a equação de regressão, com seus testes de significância, lhe forneça a teoria. Teoria, então, passa a se traduzir em mera explicação dos resultados "significantes", ou seja, daqueles coeficientes com um valor alpha menor do que 0,05 . Até mesmo a magnitude dos coeficientes gerados fica em segundo plano, quando na maior parte das vezes essa magnitude é muito mais importante do que a "significância".

Ao escolher técnicas quantitativas para uma análise sociológica, é importantíssimo não cair nessa situação descrita acima, mas realizar boas análises exploratórias antes de optar por um modelo $\mathrm{X}$ ou $\mathrm{Y}$, tendo em conta que as relações entre as variáveis construídas a partir de dados observados são bastante influenciadas pela forma da distribuição de cada uma em separado, e das distribuições conjuntas das mesmas. De uma maneira geral, antes da escolha de uma técnica estatística para analisar os dados, vale levar em consideração os seguintes passos, na ordem em que são mencionados: a) a hipótese que está sendo investigada; b) qual a teoria que está por detrás dessa hipótese e que fornece uma explicação para ela; c) que tipo de informações os dados que tenho em mãos podem me oferecer; d) de que forma eles foram coletados (e.g. amostragem representativa ou não, estratificada ou não, questionários anônimos ou não, quem foram os aplicadores, contexto da aplicação etc.); e) as distribuições de cada variável; f) as distribuições conjuntas dos pares de variáveis e as distribuições da variável dependente com as demais; g) que mecanismos a teoria introduz para relacionar os "controles" ou variáveis independentes que escolho colocar no modelo com a variável dependente. Por fim, como já foi dito, vale a pena realizar uma boa análise exploratória dos dados com métodos não paramétricos, 
correlações e cruzamento de variáveis entre outros, para conhecêlos bem antes de entrar com a análise paramétrica.

\section{A pesquisa quantitativa e a pesquisa qualitativa: uma cisão necessária?}

Muitas vezes parece haver uma cisão nas ciências sociais, não apenas no Brasil, mas em todos os Países que desenvolvem pesquisas sociológicas, entre os pesquisadores "quantitativos" e os "qualitativos". Inúmeras discussões foram e continuam sendo feitas em torno dessa divisão, tanto do ponto de vista epistemológico quanto do ponto de vista da própria definição da prática sociológica (ver, por exemplo, Cook e Reichardt 1979). Desse modo, não pretendo me aprofundar muito nos meandros dessa discussão, mas apenas oferecer alguns subsídios para a reflexão. De grosso modo, a cisão entre os pesquisadores "qualitativos" e os "quantitativos" acaba se traduzindo numa divisão entre aqueles que "gostam de matemática e estatística" e aqueles que não gostam, e leva a muitos preconceitos e rotulações, cindindo pesquisadores até mesmo do ponto de vista das correntes teóricas que uns e outros escolhem seguir e, consequentemente, dos objetos de investigação selecionados. Seria essa uma cisão realmente necessária?

É importante ter em conta que as fronteiras que dividem a pesquisa quantitativa da qualitativa não são precisas. Alguns pesquisadores colocam essa distinção até mesmo entre pesquisas que fazem o uso de dados contínuos ou categóricos. Muitos distinguem entre as pesquisas que usam técnicas estatísticas e aquelas que optam por técnicas etnográficas tais como "entrevistas não estruturadas", "análise de discurso" e "observação participante". Existem ainda aqueles que chamam de quantitativa qualquer investigação que usa de métodos de contagem de eventos (ver a introdução de Hardy and Bryman (2009) para uma discussão sobre essas definições). Assim, se as classificações são muitas, elas são também no mínimo muito imprecisas, e as delimitações de fronteiras mostram-se profundamente elásticas. Se por um lado 
| 130 |

Uma questão de método: desafios...

uma análise de discurso pode contar, tabular e classificar o uso de determinadas palavras, e uma etnografia pode também fazer uso de contagens como, por exemplo, enunciando a proporção de pessoas que possuem uma certa opinião em uma comunidade, por outro lado a realidade social é sempre multifacetada, e a quantidade de informação disponível tem que ser filtrada para análise em ambos os tipos de pesquisa. Tanto pesquisadores que se vêm como quantitativos como aqueles que se classificam como qualitativos realizam algum tipo de "redução" dos dados disponíveis a fim de empreender suas análises, o que implica em escolhas subjetivas. Concluindo, embora exista uma distinção entre metodologias qualitativas e quantitativas de pesquisa em ciências sociais, existe também uma grande zona intermediária em que as técnicas classificadas dentro de uma ou outra corrente metodológica se fundem possibilitando um conhecimento mais profundo dos objetos de pesquisa. Como indagam Hardy e Bryman (2009, p.2), "o fato de que um pesquisador calcula um coeficiente de correlação ou baseia uma conclusão em diferenças na contagem de eventos de repente atira sua pesquisa no campo quantitativo? Será que isso importa, desde que os procedimentos sejam sistemáticos e as conclusões bem fundamentadas?"18

\section{Considerações Finais}

Todos os "tipos" (a palavra "tipo" tomada entre aspas para tentar justamente evitar a rotulação) de pesquisa sociológica estão em busca de desvendar os mecanismos que podem responder a determinadas questões sobre a sociedade. Embora a maneira de apresentar o problema a ser pesquisado possa diferir, os objetivos são similares. Qualquer "tipo" de pesquisa também se volta para

\footnotetext{
${ }^{18}$ Em inglês no original: "Does the fact that a researcher calculates a correlation coefficient or bases a conclusion on differences in the counts of events suddenly toss the research into the quantitative camp? Does it matter, so long as the procedures are systematic and the conclusions are sound?"
} 
a literatura prévia no tema de análise buscando eliminar questões ou qualificar o objeto de análise de forma a produzir material original e relevante. Essa revisão bibliográfica não deve se afastar de nenhuma fonte devido ao desconhecimento pelo pesquisador do método de análise desta, sob pena de se perder informação relevante ou se repetir análises já consolidadas.

As metodologias ditas quantitativas possuem algumas vantagens em relação à validação do conhecimento que produzem em referência aos cânones científicos. Não é sem razão que Émile Durkheim as empregou magistralmente em sua obra O Suicídio ${ }^{19}$ (2000). Elas são mais dependentes das ferramentas de análise, proporcionando maior objetividade, e a importante questão do erro amostral se define com muito mais precisão na análise quantitativa. De fato, a pesquisa quantitativa já foi definida como "uma sistemática administração do erro", tanto na amostragem/ coleta quanto na análise dos dados (Hardy e Bryman 2009). Embora a abordagem quantitativa não consiga dirimir os desvios entre a amostra e a população em estudo, ela permite definir qual a probabilidade de ocorrência de determinados eventos e quais as possiblidades de confirmar uma hipótese como verdadeira sendo ela falsa. Por outro lado, as nuances da subjetividade do objeto de pesquisa nas ciências sociais, e a compreensão do sentido da ação social, podem ser muito bem captados com um conhecimento mais aprofundado desse objeto, tal como ocorre nas metodologias qualitativas.

Enfim, o principal desafio da pesquisa quantitativa em ciências sociais, a meu ver, é realizar uma pesquisa sistemática e consciente de modo a administrar o erro estatístico deixando as conclusões claras em termos de probabilidades. Deve levar em consideração para isso desde as técnicas de amostragem, coleta e classificação dos dados, os métodos estatísticos de análise, os

${ }^{19} \mathrm{O}$ que não significa que as conclusões obtidas por Durkheim estivessem corretas. Significa apenas que a metodologia estava exposta tão claramente que torna fácil a validação ou a refutação das conclusões (ver a crítica às análises quantitativas realizadas em O Suicídio por Poppel e Day, 1996). 
resultados relevantes para as conclusões a que chega o pesquisador, até e principalmente os mecanismos sociais que estão por detrás da questão investigada, e da escolha do método de investigação (pois essa escolha muitas vezes implica em assumir implicitamente determinados mecanismos sociais).

Esse desafio pode ser estendido igualmente à pesquisa qualitativa. Acredito que seria um erro, nesse momento em que cresce a demanda pelo ensino de metodologias de pesquisa em ciências sociais nas universidades brasileiras, permitir aos alunos a escolha entre as cadeiras de "métodos quantitativos" e "métodos qualitativos" de pesquisa. Ambas deveriam ser obrigatórias, pois o estudante de sociologia deve apreender os métodos de pesquisa como possíveis instrumentos do conhecimento que auxiliam na descoberta dos mecanismos que regem a ação social. Nesse sentido, uma visão mais abrangente possibilitará sem dúvida uma escolha mais informada dos seus métodos de análise, juntamente com um melhor entendimento da literatura prévia a respeito do objeto pesquisado, gerando trabalhos científicos de maior qualidade e originalidade.

\section{Referências}

AGUIAR, Neusa (org). Desigualdades Sociais, redes de sociabilidade e participação política. Belo Horizonte: Ed.UFMG, 2007

BLAU, P., and DUNCAN, O. The American Occupational Structure. New York, Wiley Books, 1967.

BECKER, H. A escola de chicago. Mana vol.2 no.2 Rio de Janeiro Oct. 1996

CANO, I. Nas trincheiras do método: o ensino da metodologia das ciências sociais no Brasil. In: Sociologias, Porto Alegre, ano 14, no 31, set./dez. 2012, p. 94-119

COOK, T., REICHARDT, C. Qualitative and quantitative methods in evaluation research. Beverly Hills: Sage, 2012. 
COLEMAN, Equality of Educational Opportunity ("The Coleman Report"), 1966.

COOKSON, P. and PERSELL, C. Preparing for Power. NewYork, Basic, 1985.

Di MAGGIO, P. e USEEM, M. "The Arts in cultural Reproduction" In: Cultural and Economic Reproduction in Education. London: Routledge \& Kegan Paul, 1982.

DURKHEIM, E. O suicidio: estudo de sociologia. São Paulo: Martins Fontes, 2000.

FREITAS, R. S. de e COLLARES, A. O modus tollens, o holismo de Duhem-Quine e as ciências sociais. In: Dados, vol.44, no.2, 2001.

FREITAS, R. S. de. A produção de pesquisa sociológica. In: Horizontes das Ciências Sociais no Brasil: sociologia. Martins, C. B e Martins, H. H. T. S. (Coordenadores). São Paulo: ANPOCS, p. 53-78, 2010.

FREITAS, H.; OLIVEIRA, M.; SACCOI, A. Z., MOSCAROLA, J. O metodo de pesquisa survey. In: Revista de Administração, São Paulo, v.35, n.3, p. 105-112, julh/set 2000.

GOULDNER, A. The Future of Intellectuals or the Rise of the New Class. New York: Seabury, 1979.

HARDY, M. e BRYMAN, A. The handbook of Data Analysis. Sage Publications, 2009.

HECKMAN, J. Sample selection bias as a specification error. In: Econometrica. Journal of the Econometric Society (47): pp.153-161, 1979.

LAREAU, A., \& WEININGER, E. Cultural Capital in Educational Research: A Critical Assessment. In: Theory and Society, 567606, 2003. 
Uma questão de método: desafios...

MARINO, J. Fundamentos do 'Paradigma Metodológico Causal' nas Ciências Sociais. In: Sociologias, Porto Alegre, ano 14, no 31, set./dez, p. 20-50, 2012.

MARTINS, C. O que é Sociologia. 28 $8^{a}$ ed. São Paulo. Ed. Brasiliense, 1982.

MARTINS, H. H. T. S. A sociologia como campo científico. In: Horizontes das Ciências Sociais no Brasil: sociologia. Martins, C. B e Martins, H. H. T. S. (Coordenadores). São Paulo: ANPOCS, p.13-23, 2010.

MERTON, R. K., Social Theory and Social Structure, Free Press, 1968.

MYERS, S. L. Why are crimes underreported? What is the crime rate? Does it really matter? Social Science Quarterly, Vol. 61 n.1, junho, 1980. Pp.23-43.

NATIS, L. Modelos Lineares Hierárquicos. Estudos em Avaliação Educacional n.23, jan-jun, 2001.

PEREIRA, M. A. M. e SIMÕES, S. A entrevista de survey como interação social: Atitudes e posição na estrutura social dos respondentes como fatores explicativos da susceptibilidade aos efeitos nas respostas. 2 v., enc.: Tese (doutorado) Universidade Federal de Minas Gerais. Faculdade de Filosofia e Ciências Humanas, 2010.

PINTO, A. S. e RIBEIRO, L. M. L. (Orgs.) A análise criminal e o planejamento operacional. Coleção Instituto de Segurança Pública. Vol. 1, Rio de Janeiro, 2008.

POPPEL, F. e DAY, L. A Test of Durkheim's Theory of Suicide without Commiting the 'Ecological Fallacy'. In: American Sociological Review, no 61, pp. 500-507, 1996.

RAUDENBUSH, S. W. e BRYK, A. Hierarchical Linear Models: applications and data analysis methods. SAGE, 2002. 
ROBINSON, R. and GARNIER, M. Class Reproduction Among Men and Women in France. In: American Journal of Sociology 91: 250-280, 1985.

SCHNEIDER, B. et al. Estimating Causal Effects using experimental and observational designs. Washington, D. C.: American Educational Research Association, 2007.

SILVA, P. L. N.; Carneiro, P. D. G.; Franca, L. M. Análise estatística de dados da PNAD: incorporando a estrutura do plano amostral. Ciência \& Saúde Coletiva, 7(4):659-670, 2002.

SIMÕES, S. e PEREIRA, M. A. M. A arte e a ciência de fazer perguntas. Aspectos cognitivos da metodologia de survey e a construção do questionário. In: Aguiar, Neuma (org). Desigualdades Sociais, redes de sociabilidade e participação política. Belo Horizonte: Ed.UFMG, 2007.

SOARES, G. A. D. O calcanhar metodológico da ciência política no Brasil. Sociologia, Problemas e Práticas. n.48, 2005, p. 27-52.

SORENSEN, A. Theoretical mechanisms and the empirical study of social processes. In: HËDSTRÖM, P. and SWEDBERG. R. Social Mechanisms: An Analytical Approach to Social Theory. Cambridge University Press, 1998.

STINCHCOMBE, A. Constructing Social Theories. Harcourt, Brace \& World, 1968. 